\title{
Diagnóstico de la dinámica cardiaca durante 16 horas desde los sistemas dinámicos aplicable en UCI
}

\author{
Diagnosis of cardiac dynamics for 16 hours from \\ dynamical systems applicable to ICU
}

Javier O Rodríguez $V^{1,2}$, Signed E Prieto B ${ }^{1,2}$, Sandra C Correa $\mathrm{H}^{1,2}$, Henry Oliveros $\mathrm{R}^{2,3}$, María Y Soracipa $\mathrm{M}^{1,2}$, Alejandro Velasco $\mathrm{R}^{1,2}$, Susana M Silva $\mathrm{C}^{1,2}$, Jairo J Jattin $\mathrm{R}^{1,2}$, Cesar Valdés $\mathrm{C}^{1,2}$, Daniela Suarez $\mathrm{G}^{1,2}$

Forma de citar: Rodríguez JO, Prieto SE, Correa SC, Oliveros H, Soracipa MY, Velasco A, et al. Diagnóstico de la dinámica cardiaca durante 16 horas desde los sistemas dinámicos aplicable en UCI. Rev Univ Ind Santander Salud. 2017; 49(1): 75-84. DOI: http://dx.doi.org/10.18273/revsal.v49n1-2017007 두요 (1)

\section{RESUMEN}

Introducción: El comportamiento caótico de la dinámica cardiaca normal y aguda ha sido caracterizado en el contexto de la teoría de los sistemas dinámicos y la geometría fractal. Objetivo: Desarrollar una nueva metodología diagnóstica para la evaluación de la dinámica cardiaca en pacientes de UCI, durante 16 horas. Metodología: Este es un estudio en pacientes ingresados a Cuidados Intensivos posquirúrgicos (UCI) y sujetos sanos, tomando un total de 47 registros electrocardiográficos continuos y/o Holter, normales y con patología aguda, evaluados en 16 horas. Se desarrolló una inducción con dos dinámicas normales y tres de UCI; a partir de los valores máximos y mínimos de la frecuencia cardiaca/hora y total de latidos/hora, registrados durante 16 horas se construyeron atractores para evaluar sus espacios de ocupación y dimensión fractal, con el fin de establecer diferencias e igualdades respecto a estados normales y patológicos. Se realizaron medidas de sensibilidad y especificidad con las dinámicas restantes para comparar el diagnostico matemático con el diagnóstico clínico. Resultados: Los espacios de ocupación de los atractores cardiacos diferencian dinámicas cardiacas normales de dinámicas con enfermedad crónica y aguda, detectando además dinámicas patológicas con valores superiores a los límites de normalidad, logrando valores de sensibilidad de 0,937 y especificidad del 1. Conclusión: se estableció una nueva metodología de evaluación de la dinámica cardiaca de utilidad para el seguimiento clínico en pacientes de UCI.

Palabras clave: fractales, matemática, dinámicas no lineales, Modelos Teóricos, Frecuencia cardíaca, Electrofisiología cardíaca

\footnotetext{
ABSTRACT

Introduction: The chaotic behavior of normal and acute cardiac dynamics has been characterized in the context of theory of dynamical systems and fractal geometry. Objective: to establish a new diagnostic method for assessing cardiac dynamics in ICU patients, for 16 hours. Methodology: This is a study in post-surgical patients admitted to

1. Clínica del Country.

2. Universidad Militar Nueva Granada. Bogotá, Colombia.

3. Hospital Militar Central. Universidad Militar Nueva Granada. Bogotá, Colombia.

Correspondencia: Javier Rodríguez Velásquez. Dirección: Cra. 79B N 51-16 Sur. Int. 5. Apto. 102, Barrio Kennedy, Bogotá D.C., Colombia. Correo electrónico: grupoinsight2025@yahoo.es. Teléfono: +7 31314057252
} 
intensive care (ICU) and in healthy subjects, taking a total of 47 continuous electrocardiographic recordings and/ or Holter, normal and acute pathology, evaluated in 16 hours. Induction with two normal and three UCI dynamics was developed; from the maximum and minimum values of the heart/time and total frequency of beats/minute for 16 hours recorded attractors they were constructed to assess their areas of occupation and fractal dimension, in order to establish differences and equalities regarding normal states and pathological. Sensitivity and specificity measurements were performed with the remaining dynamic to compare mathematical diagnosis with clinical diagnosis. Results: Space occupancy heart attractors differ dynamic normal cardiac dynamics with chronic and acute illness, in addition detecting dynamic pathological with above normal limits values, achieving sensitivity values of 0.937 and specificity of 1 . Conclusion: a new methodology for evaluating cardiac dynamics useful for clinical monitoring in ICU patients was established.

Keywords: fractals, Mathematics, Nonlinear Dynamics, Models, Theoretical, Heart Rate, Cardiac Electrophysiology.

\section{INTRODUCCIÓN}

La teoría de los sistemas dinámicos, estudia el estado y evolución de los sistemas a partir de la construcción de trayectorias que pueden ser evaluadas geométricamente en un espacio denominado espacio de fase el cual genera figuras designadas como atractores. En el espacio de fase se pueden observar tres tipos de atractores, cuya información subyacente determina el carácter predecible o impredecible del sistema ${ }^{1}$. Si se encuentran atractores que tienden a un punto o a un ciclo, son considerados predecibles, en cambio atractores con apariencia caótica o irregular son considerados impredecibles ${ }^{1}$. La geometría fractal es un tipo de geometría mediante la cual se puede calcular el grado de irregularidad de un objeto. Existen diferentes métodos para el cálculo de la dimensión fractal, que varían de acuerdo con el tipo de objeto que se medirá. La irregularidad observada en los atractores caóticos, dentro de los cuales se encuentran los atractores de la dinámica cardíaca, puede ser medida mediante el método de Box-Counting ${ }^{2-4}$.

El Ministerio de Protección Social de Colombia reportó para el año 2011 una epidemia de Enfermedades Cardiovasculares (ECV), dentro de las cuales se destaca la isquémica cardiaca o infarto considerándolas responsables del mayor número de casos fatales. El número de muertes reportadas por causa de esta enfermedad fue de 29.000 personas, en promedio 80 personas al día ${ }^{5}$. Por tal razón se ha visto la necesidad de desarrollar nuevas investigaciones con las cuales se propongan soluciones a esta problemática, tanto respecto a su diagnóstico como a su seguimiento y tratamiento.

En la literatura médica se encuentran varios métodos o técnicas para el diagnóstico del estado cardiaco, entre los que se encuentran diversas formas de cálculo de la variabilidad de la frecuencia cardiaca, cuya validación de resultados está sujeta a técnicas estadísticas, evaluando de esta manera la variabilidad mediante promedios y desviaciones estándar ${ }^{6}$. Se ha encontrado que mediante estos métodos se pueden establecer relaciones entre diferentes medidas y algunas alteraciones patológicas, sin embargo, no es posible prever qué pacientes presentarán muerte cardiaca súbita o infarto agudo de miocardio para cada caso específico.

Investigaciones desarrolladas en el contexto de la teoría de los sistemas dinámicos, hacen evidente que el sistema cardiovascular presenta un comportamiento no lineal ${ }^{7}$, es decir que se trata de un sistema que no obedece a un movimiento senoidal. Por ejemplo, Ritzenberg, et al. ${ }^{8}$ demostraron la no linealidad en el electrocardiograma. Mediante el análisis de la Variabilidad de la Frecuencia Cardiaca (VFC) revelaron la no linealidad del ritmo cardiaco, presente en pacientes con alto riesgo de muerte cardiaca súbita. Incluso, se encontró una asociación entre la pérdida de la complejidad de la variabilidad fisiológica con la reducción de la dinámica del ritmo cardiaco antes de la muerte súbita y el envejecimiento-11. Así mismo, medidas fractales en pacientes con fracción de eyección menor al $35 \%$ después de infarto agudo de miocardio permitieron establecer predictores de mortalidad con mayor capacidad de discriminación que los logrados convencionalmente ${ }^{12}$.

Recientemente se han desarrollado nuevas metodologías que permiten establecer diagnósticos de aplicabilidad clínica y evaluar estados adversos del paciente, facilitando de este modo la toma de decisiones para su correcto seguimiento. El establecimiento de rangos de valores numéricos que caracterizan qué tan alejada o cercana se encuentra la dinámica cardiaca a un estado agudo, permite discernir el nivel de gravedad de las alteraciones detectadas ${ }^{13-15}$. Por ejemplo, para la evaluación de la dinámica cardiaca en 21 horas se ha desarrollado una metodología diagnóstica a partir del método de Box Counting, basada en la superposición de rejillas cuadradas para el conteo de los cuadros ocupados por el atractor cardíaco; este procedimiento permite 
tener una magnitud de las diferencias en el tamaño de los atractores, de acuerdo a si tienen más o menos cuadros ocupados, con lo cual fue posible establecer diferencias cuantitativas entre normalidad y enfermedad aguda ${ }^{16}$. La aplicabilidad y reproductibilidad de la metodología, ha sido confirmada mediante una prueba diagnóstica realizada en 150 registros Holter, hallando valores de sensibilidad y especificidad del $100 \%{ }^{16}$ y un coeficiente Kappa de uno. Debido a la abstracción de factores causales, la metodología puede evaluar los valores de la Frecuencia cardiaca (FC) tanto de registros Holter como registros electrocardiográficos continuos, debido a que solo hace uso de los valores de la FC mínima y máxima cada hora, así como el número de latidos, para establecer un diagnóstico cuantitativo de aplicación clínica.

Recientemente a partir de esta metodología se realizó un trabajo del cual se hizo una presentación preliminar en el X Congreso de Medicina Crítica y Cuidado Intensivo, en el que se logró establecer la capacidad diagnóstica de la evaluación de la ocupación espacial del atractor tomando información de solamente 16 horas. Este nuevo método diagnóstico permitiría un seguimiento más efectivo de pacientes en la UCI, al disminuir costos y recursos a nivel clínico ${ }^{17}$.

\section{OBJETIVO}

El propósito de la presente investigación es confirmar los resultados anteriores, realizando un proceso inductivo y su posterior confirmación mediante un estudio ciego con un mayor número de pacientes, desarrollando de este modo un nuevo método diagnóstico a partir de la evaluación de la dinámica cardiaca en 16 horas.

\section{METODOLOGÍA}

\section{Definiciones:}

Espacio de fases: representación gráfica de la dinámica de un sistema en un espacio de dos o más dimensiones, a partir de la ubicación de pares ordenados de una variable dinámica en el tiempo, generando un atractor ${ }^{1}$.

Dimensión fractal de Box-Counting: es una medida adimensional, calculada a partir de la siguiente fórmula ${ }^{18}$ :

$D=-\frac{\log N_{1}\left(2^{-(j+1)}\right)-\log N_{2}\left(2^{-j}\right)}{\log 2^{j+1}-\log 2^{j}}=\log _{2}-\frac{N_{1}\left(2^{-(j+1)}\right)}{N_{2}\left(2^{-j}\right)}$

Siendo: $D$ : Dimensión fractal; $N$ : Número de cuadros ocupado por el objeto; donde $N_{1}$ : número de cuadros ocupados por el objeto en la rejilla de partición de $2^{-(j+1)}$ y $N_{2}$ : número correspondiente a la partición de $2^{-j} ;$ y $j$ : Grado de partición de la cuadrícula.

\section{Población}

Se realizó un estudio que incluyó 15 dinámicas de sujetos normales provenientes de bases de datos de investigaciones previas del Grupo Insight, y 32 dinámicas con enfermedad aguda, procedentes de pacientes críticos que fueron ingresados a la UCI Postquirúrgica del Hospital Militar Central. La población fue escogida con base en una reinterpretación de la concepción normalidad-enfermedad de los sistemas dinámicos ${ }^{11}, \mathrm{y}$ en la metodología desarrollada previamente ${ }^{16}$ en la que se logra diferenciar normalidad de enfermedad a partir del número de espacios ocupados del atractor de la dinámica cardiaca. También fue escogida porque puede representar y ser comparada con cualquier tipo de dinámica de acuerdo a sus características matemáticas. Por ser una metodología física y matemática está basada en un proceso inductivo en el que a partir de pocos casos se hallan los parámetros físico-matemáticos diferenciadores que posteriormente permitirán aplicar la metodología al resto de casos.

Los diagnósticos clínicos fueron establecidos de acuerdo con el criterio de un cardiólogo experto, siguiendo los parámetros clínicos convencionales. De estos sujetos, se tomaron dos normales y tres pacientes de UCI para el desarrollo de la inducción, mientras que la información clínica de los 42 Holter restantes fue enmascarada para realizar un estudio ciego de confirmación de la inducción.

\section{Procedimiento}

Inicialmente se desarrolló una inducción a partir de dos dinámicas normales y tres pacientes de UCI. Se realizó un registro de la dinámica cardiaca durante mínimo 16 horas mediante registro Holter en el caso de los sujetos normales, y registros electrocardiográficos continuos (monitores marca Dräger) en el caso de los pacientes de la Unidad de Cuidados Intensivos Postquirúrgicos del Hospital Militar Central.

Para el desarrollo de la inducción matemática, se tomaron dos sujetos normales y tres pacientes de la UCI los cuales representaban un rango amplio de edades, de los 21 a los 91 años, así como un rango amplio de valores mínimos, máximos de latidos/hora, y del total de latidos de cada hora de la FC, también se escogieron por ser sujetos de importancia clínica ya sea esta por su condición clínica normal, crónica o aguda. A partir de estos casos se realizó la inducción matemática, para 
lo cual se tomaron los valores de frecuencia cardiaca máxima y mínima cada hora, así como el total de latidos para cada hora, para cada sujeto. En el caso de los sujetos normales, los datos fueron tomados directamente de los reportes del Holter, mientras que para los pacientes de UCI, se sistematizaron los valores de la frecuencia cardiaca registrados minuto a minuto en los monitores Dräger, para luego hallar los valores máximos y mínimos de $\mathrm{FC} /$ hora y total de latidos de la FC para cada hora.

Seguidamente, estos valores fueron utilizados para generar una secuencia de frecuencias cardiacas para construir un mapa de retardo con base en la información sistematizada (ver definiciones). Para cada uno de los atractores se superpusieron dos rejillas de 5 y 10 lat $/ \mathrm{min}$, denominadas $\mathrm{Kp}$ y $\mathrm{Kg}$ respectivamente, realizando con cada una de ellas el conteo de espacios de ocupación y el cálculo de la dimensión fractal.

Finalmente se comparó el diagnóstico físico-matemático con el diagnóstico clínico convencional, comparando las variaciones en los espacios de ocupación de la FC respecto a rangos establecidos previamente para estados de normalidad, enfermedad crónica o aguda, de acuerdo con estudios previos, estableciendo el comportamiento matemático característico de cada estado desde esta metodología.

A continuación, se desenmascaró el diagnóstico clínico de cada uno de los registros Holter y/o electrocardiográficos continuos. Se tomaron las dinámicas con diagnóstico clínico de normal o con enfermedad aguda, y se analizaron mediante el mismo procedimiento que los registros de la inducción, determinando así su diagnóstico matemático. Posteriormente se calculó la sensibilidad y especificidad asumiendo como patrón de oro el diagnóstico clínico convencional para determinar los valores de sensibilidad, y especificidad, de manera que los verdaderos positivos (VP) están representados por el número de pacientes diagnosticados clínicamente y por medio del diagnóstico matemático con enfermedad aguda, mientras que los falsos positivos (FP) son el número de registros Holter y/o electrocardiográficos continuos que matemáticamente se comportan como estudios de enfermedad aguda y cuyo diagnóstico clínico es dentro de límites normales, falsos negativos (FN) corresponde al número de registros Holter y/o electrocardiográficos continuos cuyos valores matemáticos corresponden a normalidad pero cuyo diagnóstico clínico corresponde a pacientes con enfermedad aguda y finalmente verdaderos negativos $(\mathrm{VN})$ corresponde al número de registros diagnosticados clínicamente como normales y cuyos valores matemáticos también corresponden a normalidad.
En este trabajo no se calculan intervalos de confianza, debido al tipo de diseño metodológico aplicado, que al estar sustentado en un razonamiento inductivo físicomatemático, busca establecer diferencias que deben confirmarse en cualquier caso particular. El cálculo de los valores de sensibilidad y especificidad se aplicó a todos los casos normales y con enfermedad aguda, excluyendo los casos con enfermedad crónica, debido a que la evaluación actual del Holter presenta falencias, lo que puede dar lugar a alteraciones subdiagnosticadas. En este orden de ideas, hemos tomado el diagnóstico convencional del Holter como Gold Standard para los casos normales y agudos, sin embargo, los casos crónicos requieren una validación con exámenes adicionales, lo que excede el objetivo de la presente investigación.

\section{RESULTADOS}

La Tabla 1 muestra los valores calculados de 20 de las 47 dinámicas cardiacas evaluadas, 10 dinámicas cardíacas normales y 10 de la UCI. Se seleccionaron estos 20 pacientes de la totalidad, debido a que los valores de las dinámicas cardíacas restantes tienen valores similares o cercanos a los mostrados en la Tabla 1. Los datos sombreados en gris corresponden a las cinco dinámicas tomadas para la inducción matemática.

Los dos registros normales tomados para la inducción matemática presentaron valores de 76 y 116 para la rejilla kg y de 263 y 455 para la rejilla $\mathrm{Kp}$, y dimensiones fractales de 1,79099148 y 1,97174174. Los registros provenientes de la UCI tomados para la inducción matemática presentaron valores de dimensión fractal entre 1,169925 y 1,86624861 ; los valores de ocupación en la rejilla $\mathrm{Kg}$ estuvieron entre 16 y 399 y en la rejilla Kp se encontraron entre 36 y 1439.

Las dimensiones fractales de los registros Holter evaluados en 21 horas con diagnóstico clínico normal variaron entre 1,66 y 1,94 mientras que para los registros provenientes de la UCI variaron entre 1,55 y 1,92. Los espacios ocupados por los atractores normales evaluados en 21 horas, al superponer la rejilla Kp oscilaron entre 254 y 448, para la rejilla Kg entre 73 y 119; para los pacientes de UCI la superposición de la rejilla $\mathrm{Kp}$, oscilo entre 54 y 1456; y entre 16 y 390 para la rejilla $\mathrm{Kg}$, Tabla 1.

En cuanto a los registros evaluados en 16 horas mediante la presente metodología, las dimensiones fractales de los 10 registros Holter con diagnóstico clínico normal se encontraron entre 1,69 y 1,97 ; y entre 1,1699 y 1,9509 los provenientes de la UCI. 
El comportamiento de los atractores cardíacos de dos de los pacientes provenientes de la UCI se puede ver en las Figuras 1 y 2. El cálculo de los espacios ocupados por los atractores normales al superponer la rejilla $\mathrm{Kp}$ oscilaron entre 255 y 455 , y para la rejilla Kg entre 69 y 116; para los casos de UCI la superposición de la rejilla $\mathrm{Kp}$, osciló entre 36 y 1439, y para la rejilla Kg entre 16 y 399 .

TABLA 1. Medidas de los espacios ocupados por los atractores al superponer las dos rejillas en los atractores evaluados durante 16 horas. Los 10 primeros corresponden a casos normales y los 10 últimos corresponden a dinámicas de pacientes en UCI. Kp: es la rejilla con cuadros pequeños. Kg: rejilla con cuadros grandes. Df: dimensión fractal.

\begin{tabular}{|c|c|c|c|c|}
\hline \multirow[t]{2}{*}{ No } & \multirow[t]{2}{*}{ Diagnóstico clínico } & \multicolumn{3}{|c|}{$\begin{array}{l}\text { Diagnóstico } \\
\text { matemático }\end{array}$} \\
\hline & & $\mathbf{K g}$ & Kp & Df \\
\hline 1 & Estudio dentro de los límites normales & 84 & 281 & 1,7421 \\
\hline 2 & Estudio dentro de los límites normales & 93 & 301 & 1,6945 \\
\hline 3 & Estudio dentro de los límites normales & 75 & 255 & 1,7655 \\
\hline 4 & Estudio dentro de los límites normales & 106 & 354 & 1,7397 \\
\hline 5 & Estudio dentro de los límites normales & 81 & 289 & 1,8351 \\
\hline 6 & Estudio dentro de los límites normales & 88 & 338 & 1,9414 \\
\hline 7 & Estudio dentro de los límites normales & 116 & 455 & 1,9717 \\
\hline 8 & Estudio dentro de los límites normales & 85 & 312 & 1,8760 \\
\hline 9 & Estudio dentro de los límites normales & 69 & 266 & 1,9468 \\
\hline 10 & Estudio dentro de los límites normales & 76 & 263 & 1,7910 \\
\hline 11 & Sepsis tejidos blandos. enfermedad pulmonar crónica con exacerbación no especificada. & 37 & 140 & 1,9198 \\
\hline 12 & $\begin{array}{l}\text { Epoc. Trauma antebrazo derecho al caer sobre este con el peso del cuerpo, desde su nivel posterior a } \\
\text { episodio de vértigo, se asocia HTA con controlada x falta de medicación, Insuficiencia respiratoria aguda } \\
\text { hipoxemia } \\
\text { Edema pulmonar } \\
\text { Insuficiencia cardiaca congestiva descompensada. }\end{array}$ & 93 & 316 & 1,7646 \\
\hline & $\begin{array}{l}\text { Paciente con Sepsis de Origen pulmonar. Paraclinicos: hemograma: leucos: 16600; gases arteriales: PM } \\
7,29 \text {. }\end{array}$ & & & \\
\hline 13 & $\begin{array}{l}\text { Diagnóstico de neumonía severa asociada a los cuidados de la salud basal derecha más derrame pleural } \\
\text { bilateral. Falla ventilatoria secundaria. Tep probabilidad alta wellsg. EPOC exagerado anthonisen. Falla } \\
\text { cardiaca fevi no conocida. Día } 1\end{array}$ & 399 & 1439 & 1,8506 \\
\hline 14 & $\begin{array}{l}\text { Sepsis de Origen pulmonar. Paraclinicos: hemograma: leucos: } 16600 \text {; gases arteriales: PM } 7,29 \text {. Dx: } \\
\text { neumonia severa asociada a los cuidados de la salud basal derecha }+ \text { derrame pleural bilateral. Falla } \\
\text { ventilatoria secundaria. Tep probabilidad alta wellsg. EPOC axagerado anthonisen. Falla cardiaca fevi no } \\
\text { conocida. Día } 2\end{array}$ & 359 & 1388 & 1,9510 \\
\hline 15 & $\begin{array}{l}\text { Insuficiencia renal no especificada } \\
\text { Otros signos y síntomas que involucran la función cognoscitiva y la conciencia y las no especificadas. } \\
\text { Insuficiencia respiratoria aguda }\end{array}$ & 16 & 36 & 1,1699 \\
\hline 16 & Enfermedad de vesícula, no especificada & 194 & 711 & 1,8738 \\
\hline 17 & Enfermedad de vesícula, no especificada & 217 & 837 & 1,9475 \\
\hline 18 & $\begin{array}{l}\text { Hemorragia alveolar difusa, choque séptico, Neumonía en paciente inmunosuprimido, Pneunositosis } \\
\text { pulmonar aguda-hipotemico, Doble lesión valvular mitral, insuficiencia aortica mínima, insuficiencia } \\
\text { tricupidea severa, Lupus erimatososistemico, serosistis: derrame pericardio mínimo sin repercusión } \\
\text { hemodinámica, hipertensión pulmonar severa tipo A. Antecedentes de hemorragia alveolar hace } 4 \text { años. }\end{array}$ & 27 & 85 & 1,6545 \\
\hline \multirow{3}{*}{19} & $\begin{array}{l}\text { Insuficiencia cardiaca congestiva } \\
\text { Choque cardiogenetico, }\end{array}$ & 48 & 175 & 1,8662 \\
\hline & $\begin{array}{l}\text { Falla cardiaca terminal fevi } 15 \% \\
\text { Cardiomiopatia dilatada }\end{array}$ & & & \\
\hline & $\begin{array}{l}\text { Trombofilia primaria } \\
\text { Usuario de cardio resincronizador }\end{array}$ & & & \\
\hline 20 & $\begin{array}{l}\text { Fibrilación y aleteoauricular. Paciente con hipertensión arterial y antecedentes poco claros de arritmia } \\
\text { cardiaca, dolor precordial opresivo constante. Intensidad } 7 / 10 \text { EVA irradiada región interescapular } \\
\text { asociada a disnea, palpitaciones diaforesis, náuseas y emesis. Fue valorada en urgencias, gasto cardiaco, } \\
\text { falla respiratoria realizada inrubación orotraqueal solicitando enzimas cardiacas con reporte de curva } \\
\text { positiva inicia soporte vasoactivo para mantener adecuadas presiones de perfusión. Solicitan traslado a } \\
\text { UCI para monitoreo y manejo hemodinámico. }\end{array}$ & 32 & 100 & 1,6439 \\
\hline
\end{tabular}




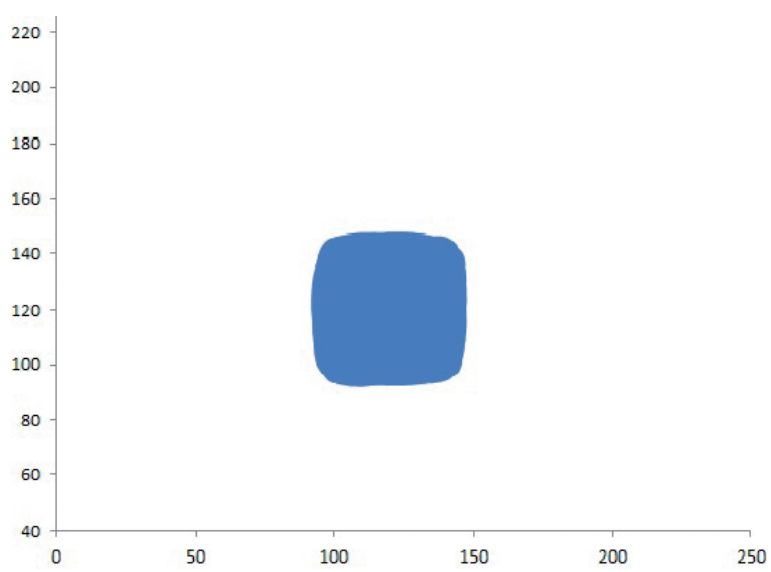

FIGURA 1. Atractor correspondiente a la dinámica del paciente No 11, con sepsis en tejidos blandos y enfermedad pulmonar crónica con exacerbación no especificada. Se puede observar una ocupación espacial de 140 en la rejilla Kp, menor al rango de normalidad.

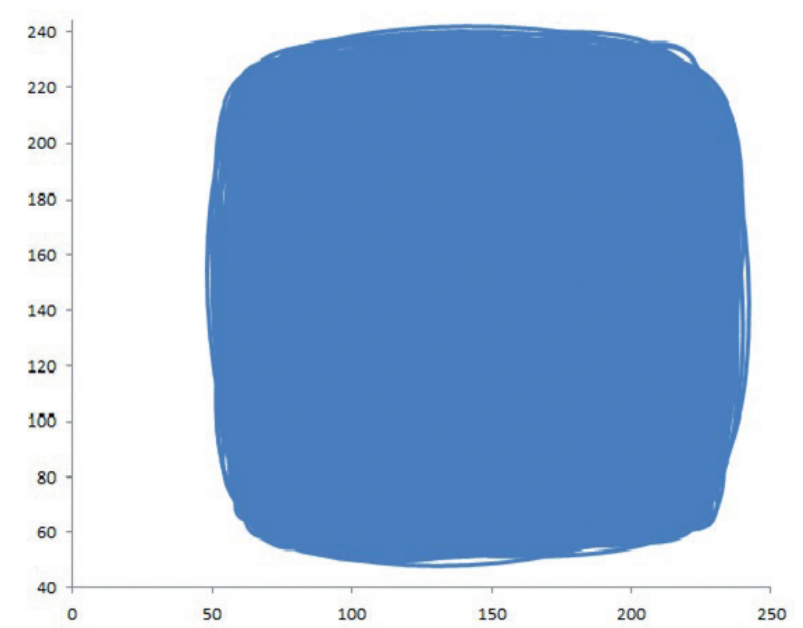

FIGURA 2. Atractor correspondiente a la dinámica del paciente No 13, con falla cardiaca, donde se evidencia una ocupación espacial superior a los valores normales, que corresponde a 1439 cuadros en la rejilla Kp.

\section{Resultado de ayuda diagnóstica}

Con base en los resultados hallados en la rejilla $\mathrm{Kp}$, se estableció que la normalidad se encuentra entre 210 y 534. Valores entre 209 y 74 corresponden a enfermedad crónica (ver figura 1) y valores inferiores a 74 corresponden a enfermedad aguda. Además, se estableció otro rango asociado a enfermedad, correspondiente a valores iguales o mayores a 535 .

De acuerdo con este diagnóstico físico-matemático, se encontró que dos dinámicas de pacientes de la UCI presentaron valores correspondientes a normalidad, mientras que 16 presentaron valores correspondientes a enfermedad crónica y cuatro enfermedad aguda.
Adicionalmente, se encontró que hubo 10 dinámicas que presentaron valores por encima del límite superior de normalidad.

$\mathrm{Al}$ evaluar las dinámicas normales y con enfermedad se encontraron valores de sensibilidad de $0,937 \mathrm{y}$ especificidad del 1.

\section{DISCUSIÓN}

Este es el primer trabajo en el que se establece una nueva metodología desarrollada en el contexto de la teoría de los sistemas dinámicos y la geometría fractal, para diagnosticar la dinámica cardiaca de sujetos normales y pacientes de la UCI durante 16 horas mediante los registros electrocardiográficos continuos y/o Holter, a partir de la cuantificación de los espacios de ocupación de atractores cardiacos a partir del método de Box Counting. Los resultados hallados muestran que es posible establecer diferencias específicas para la UCI, cuantitativas y reproducibles, entre dinámicas cardiacas normales, con enfermedad crónica y con enfermedad aguda, estableciendo además un rango de enfermedad caracterizado por valores superiores a los valores más altos de normalidad, evidenciando que la normalidad se ubica en un rango intermedio entre valores muy altos o muy bajos de estados patológicos. Se encontraron valores de sensibilidad de 0,937 y especificidad del 1 , al diferenciar dinámicas normales y enfermas. Desde esta perspectiva, la metodología puede ser la base de futuros dispositivos que codifiquen la señal de la frecuencia cardiaca monitorizada en la UCI, optimizando el seguimiento continuo de la evolución del paciente.

Anteriormente se había establecido que a partir de los espacios de ocupación de los atractores cardiacos era posible establecer diferencias entre normalidad y enfermedad aguda, tanto en 21 horas ${ }^{16}$ como en $16^{17}$. Estos trabajos evidenciaron que era posible establecer un rango de normalidad entre valores de 200 a 478 en la rejilla $\mathrm{kp}$, mientras que la enfermedad aguda se encontraba por debajo de 74, y la enfermedad crónica solía presentar valores entre 74 y 200. Por otro lado, los trabajos desarrollados por Goldberger, et al. ${ }^{11}$, contradijeron las nociones del principio de homeostasis, al revelar que el sistema cardiaco enfermo, se asocia a comportamientos altamente regulares o irregulares, mientras que la normalidad se establece como un comportamiento intermedio entre dichos extremos. Los resultados del análisis de las dinámicas de los pacientes de la UCI indican que se pueden presentar valores físicomatemáticos tanto de normalidad, como de enfermedad crónica o enfermedad aguda. Sin embargo, solamente dos 
pacientes presentaron valores asociados a normalidad. Por otro lado, las dinámicas con valores superiores a los valores de normalidad pueden asociarse a casos con intervenciones terapéuticas como inotrópicos, que aceleran el ritmo cardiaco, lo que implica un aumento en la ocupación espacial del atractor. Por ejemplo, las dinámicas 13 (ver Figura 2) y 14 corresponden a un paciente que presento Falla cardiaca; los valores matemáticos están muy por encima de los límites de normalidad, evidenciando una alteración patológica. A diferencia del trabajo de Goldberger, donde se señala un comportamiento general de la dinámica cardiaca, en la que la normalidad corresponde a un estado intermedio entre valores patológicos, pero no se cuenta con valores específicos para establecer un diagnóstico clínico ${ }^{11}$, en el presente trabajo se evidencia que efectivamente la normalidad es un estado intermedio entre dos extremos patológicos y la dinámica crónica, logrando establecer y predecir valores cuantitativos específicos para los rangos de normalidad y enfermedad, lo que permite su aplicación clínica.

La validez del estudio se sustenta en el tipo de metodología del cual parte. Al tratarse de un método basado en el tipo de razonamiento inductivo de la física teórica, con pocos casos es posible llegar a afirmaciones de tipo universal, pues se está evidenciando un orden matemático subyacente al proceso de salud-enfermedad de la dinámica cardíaca, al margen de la etiología de la patología, caracterizado por una variación progresiva del número de espacios ocupados por el atractor caótico. Así, se muestra una auto-organización matemática subyacente al fenómeno, aplicable a cualquier caso particular. Con el fin de sustentar estos hallazgos a nivel clínico de una forma más específica pueden realizarse futuros estudios siguiendo las guías STARD para informes de estudios precisión diagnóstica.

Las señales eléctricas del corazón son reproducidas de manera gráfica mediante la electrocardiografía, que es una herramienta ampliamente utilizada para detectar alteraciones en el ritmo cardiaco ${ }^{17}$. La monitorización continua puede ser hecha mediante un Holter o con los monitores que se utilicen en los distintos escenarios de la $\mathrm{UCI}^{17}$. A finales del siglo pasado el análisis de la variabilidad de la frecuencia cardiaca, fue establecido como herramienta predictora de mortalidad específicamente en el caso de pacientes con infarto agudo de miocardio. Con el paso del tiempo fue empleado para estratificación de riesgo en pacientes cursando con enfermedad cardiovascular; sin embargo, aún no es posible prever desde estas herramientas que dinámica cardiaca entrará a paro cardiaco o muerte cardiaca súbita, o aún alertar con horas de antelación estos desenlaces. Es así que se han incorporado conceptos matemáticos provenientes de la teoría del caos y geometría fractal ${ }^{18}$ tal como es planteado por investigadores en un estudio realizado en población geriátrica ${ }^{19}$. Sin embargo, la aplicabilidad clínica de estas metodologías aún es discutida ${ }^{20}$. También se han empleado métodos dinámicos no lineales en series de electrocardiogramas de corta duración y se ha establecido que estos métodos podrían complementar el diagnóstico electrocardiográfico, demostrando la aplicabilidad de la teoría de caos y las dinámicas no lineales en la dinámica cardiovascular ${ }^{21}$. Es menester resaltar que la metodología aplicada para el presente estudio, puede diagnosticar cualquier caso particular, identificando los cuatro estados dinámicos encontrados, permitiendo un seguimiento más estricto de los procesos de la dinámica cardiaca del paciente en el tiempo a partir de los atractores cardiacos, de gran utilidad para el seguimiento del paciente crítico de la UCI, corroborando hallazgos de un trabajo previo ${ }^{17}$. Con esta metodología en futuros trabajos se podría comparar el comportamiento de la dinámica cardiaca evaluada mediante este método con el análisis de otras variables hemodinámicas, especialmente en los rangos en los que se presentan valores característicos de alteración patológica, ya sea por encima o por debajo de los valores límites de normalidad, con el fin de establecer posibles predicciones de mortalidad superando barreras evidenciadas en trabajos anteriores realizados donde es discutida la aplicabilidad clínica o donde los tiempos de evaluación son menores.

La metodología desarrollada aplicada en este estudio está basada en el método de la física teórica ${ }^{22}$ en el que a partir de la aplicación de leyes y principios físico matemáticos se toma un fenómeno complejo y se simplifica, permitiendo de esta forma estudiar sistemáticamente el problema y tener una comprensión general, el proceso generalmente utilizado para esto es la inducción, en la que a partir de pocos casos se hallan las características determinantes del fenómeno que logre simplificarlo y hacerlo comprensible, permitiendo además desarrollar metodologías que sean aplicables a todos los casos, de tal forma que este método y sus conclusiones no dependen del número de casos que se toman, ni de otros parámetros estadísticos o poblacionales como sesgos, tamaño de muestra, etc. En este trabajo y en la metodología desarrollada previamente ${ }^{16}$ se desarrolló este mismo proceso, lo que ha llevado a una simplificación del fenómeno permitiendo establecer la aplicación de la metodología no solo para 21 horas $^{16}$ sino también para 16 horas $^{17}$. 
El método de la física teórica es muy diferente al actualmente seguido en la medicina, en el cual el tamaño de la muestra si puede llegar a alterar significativamente las conclusiones obtenidas, lo que difícilmente permite desarrollar generalizaciones que puedan ser aplicables a cualquier caso, es por esto que en este trabajo se busca mostrar una vía para el desarrollo de generalizaciones en la medicina.

Continuando con trabajos desarrollados para evaluar la dinámica cardiaca del adulto desde esta perspectiva de investigación físico-matemática, se desarrolló una metodología basada en una ley matemática de aplicación clínica a partir de la cual se pueden deducir y evaluar todas las posibles dinámicas cardiacas, caracterizando dinámicas cardiacas normales, agudas y en evolución ${ }^{15}$. Recientemente, la metodología evaluó la dinámica cardiaca neonatal, logrando predecir alteraciones cardiacas asociadas a sepsis tres y seis horas antes de presentarse el episodio de sepsis ${ }^{23}$. Recientemente se evaluó la aplicabilidad clínica y la reproductibilidad de esta metodología mediante un estudio ciego con 115 registros Holter, hallado valores de sensibilidad y especificidad del $100 \%$; también se desarrolló un trabajo en el que se confirmó su aplicabilidad a casos con diagnóstico de arritmia ${ }^{24}$. El presente trabajo amplía la aplicación de los sistemas dinámicos a la clínica.

Otra metodología fundamentada en la teoría la probabilidad y las proporciones de entropía, predice diferencias cuantitativas entre normalidad, enfermedad crónica, enfermedad aguda y evolución entre estos $\operatorname{estados}^{13}$. Se confirmó la aplicabilidad clínica en diferentes pruebas diagnósticas mediante estudios estadísticos, en 300, 450, 600, dinámicas cardiacas normales y patológicas ${ }^{25}$ logrando en todos los casos valores de sensibilidad y especificidad de $100 \%$, demostrado su utilidad en el diagnóstico de la dinámica cardiaca en pacientes de la $\mathrm{UCI}^{26}$, después de las intervenciones, siendo un diagnóstico independiente de los parámetros diagnósticos utilizados actualmente, y logrando además detectar procesos de agudización subdiagnosticados.

En analogía a las teorías físicas acausalistas que sustentan la física moderna ${ }^{27,28}$, la presente metodología logra establecer cuantificaciones objetivas y reproducibles aplicables a cada caso particular independientemente de consideraciones epidemiológicas y estadísticas, desde una perspectiva a causal desarrollado diagnósticos y predicciones en áreas como la inmunología ${ }^{29}$, la epidemiología ${ }^{30}$, la morfometría de diferentes estructuras macroscópicas y microscópicas que componen el cuerpo humano ${ }^{31-33}$, y la infectología ${ }^{34}$, evidenciando órdenes físico-matemáticos subyacentes a los diferentes fenómenos médicos.

\section{AGRADECIMIENTOS}

Este trabajo hace parte de los productos alcanzados para el protocolo C025-2014, financiado por el fondo de investigaciones del Hospital Militar Central. Extendemos nuestros agradecemos a la Doctora Luz Ávila, Jefe Unidad de Investigación Científica y al Coronel Medico Luis Castro, Subdirector de Docencia e Investigación Científica. También agradecemos a Jaime Sánchez por su apoyo.

De igual manera agradecemos a las directivas de la Universidad Militar Nueva Granada y a la facultad de medicina por el apoyo dado a nuestras investigaciones y especialmente al Semillero, Línea de Profundización e Internado Especial: Física y Matemáticas Aplicadas a la Medicina, gracias al cual los estudiantes de la Facultad de Medicina fueron partícipes de esta investigación.

Al Centro de Investigaciones de la Clínica del Country, en especial al Dr. Tito Tulio Roa, Director de Educación Médica, Dr. Jorge Ospina, Director Médico, Dr. Alfonso Correa, Director del Centro de Investigaciones, y a las Doctoras Adriana Lizbeth Ortiz, Epidemióloga, y Silvia Ortiz, Enfermera Jefe del Centro de Investigaciones, así mismo a la enfermera Sandra Rodríguez y a los Bacteriólogos Camilo Benítez y Juan Alexander Rojas.

\section{CONSIDERACIONES ÉTICAS}

Este trabajo tiene como fundamentos los principios éticos para las investigaciones médicas en seres humanos de la declaración de Helsinki de la Asociación Médica Mundial, el código de Nuremberg y el reporte de Belmont, y cumple en general con las normas éticas, científicas, técnicas y administrativas para la investigación en salud, basada en la resolución No. 008430 de 1993, y específicamente con el título 11 referente a la investigación en seres humanos, al estar clasificado en la categoría de investigación sin riesgo, dado a que se hacen cálculos sobre resultados de exámenes previamente prescritos sin ninguna intervención directa sobre los pacientes. Por esta razón, cuenta con la aprobación del Comité de Ética en Investigación del Hospital Militar Central, de acuerdo con el acta No. 19696 del 13 de julio de 2015.

\section{CONFLICTOS DE INTERÉS}

Los autores declaran no tener conflictos de interés. 


\section{REFERENCIAS}

1. Devaney R. A first course in chaotic dynamical systems theory and experiments. Reading Mass: Addison- Wesley; 1992.

2. Mandelbrot B. The Fractal Geometry of Nature. Barcelona: Freeman Tusquets Eds S.A.; 1972: p.317.

3. Falconer K. Fractal geometry. Mathematical Foundations and Applications. New York: John Wiley \& Sons Ltd; 2003: p.41-43.

4. Peitgen H. Strange attractors, the locus of chaos. En: Chaos and Fractals: New Frontiers of Science. New York: Springer-Verlag; 1992: p.655-768.

5. Ministerio de Salud y Protección Social. Colombia enfrenta epidemia de enfermedades cardiovasculares y diabetes. Boletín de Prensa No 077 de 2014.

6. Gallo J, Farbiarz J, Alvarez D. Análisis espectral de la variabilidad de la frecuencia cardíaca. Iatreia. 1999; 12(2): 61-71.

7. Goldberger A, Rigney D, West B. Chaos and fractals in human physiology. Sci Am. 1990; 262: 42-49.

8. Ritzenberg AL, Adam DR, Cohen RJ. Period multupling-evidence for nonlinear behaviour of the canine heart. Nature. 1984; 307(5947): 159-161.

9. Goldberger AL, West BJ. Applications of nonlinear dynamics to clinical cardiology. Ann N Y Acad Sci. 1987; 504: 195-213.

10. Goldberger AL, Rigney DR, Mietus J, Antman EM, Greenwald S. Nonlinear dynamics in sudden cardiac death syndrome: heart rate oscillations and bifurcations. Experientia. 1988; 44(11-12): 983987.

11. Goldberger A, Amaral L, Hausdorff J, Ivanov P, Peng C, Stanley H. Fractal dynamics in physiology: alterations with disease and aging. Proc Natl Acad Sci USA. 2002; 99(Suppl 1): 2466-2472. DOI: 10.1073/pnas.012579499.

12. Huikuri HV, Mäkikallio TH, Peng CK, Goldberger AL, Hintze U, Møller M, et al. Fractal correlation properties of R-R interval dynamics and mortality in patients with depressed left ventricular function after and acute myocardial infarction. Circulation 2000; 101(1): 47-53.

13. Rodríguez J, Prieto S, Domínguez D, Melo M, Mendoza F, Correa C, et al. Mathematical-physical prediction of cardiac dynamics using the proportional entropy of dynamic systems. J Med Med Sci. 2013; 4(8): 370-381.

14. Rodríguez J, Correa C, Ortiz L, Prieto S, Bernal $\mathrm{P}$, Ayala J. Evaluación matemática de la dinámica cardíaca con la teoría de la probabilidad. Rev Mex Cardiol. 2009; 20(4):183-189.
15. Rodríguez, J. Mathematical law of chaotic cardiac dynamics: Predictions for clinical application. J Med Med Sci. 2011; 2(8): 1050-1059.

16. Rodríguez J, Prieto S, Bernal P, Soracipa Y, Salazar $\mathrm{G}$, Isaza $\mathrm{D}$, et al. Nueva metodología de ayuda diagnóstica de la dinámica geométrica cardíaca: dinámica cardíaca caótica del Holter. Rev Acad Colomb Cienc. 2011; 35(134): 5-12.

17. Rodríguez J, Prieto S, Correa C, Oliveros H, Soracipa Y, Amaya J, et al. Sistemas dinámicos aplicados a la disminución del tiempo de diagnóstico de la dinámica cardíaca de 24 a 16 horas en holter y registros electrocardiográficos continuos. X Congreso de Medicina Crítica y Cuidado Intensivo. Cartagena, Colombia. 2015.

18. Peitgen O, Jürgens H, Dietmar S. Chaos and fractals: new frontiers of science. New York: SpringerVerlag; 1992: p.192-94.

19. Nicolini P, Ciulla MM, De Asmundis C, Magrini F, Brugada $\mathrm{P}$. The prognostic value of heart rate variability in the elderly, changing the perspective: from sympathovagal balance to chaos theory. Pacing Clin Electrophysiol. 2012; 35(5): 622-638. DOI: $10.1111 / \mathrm{j} .1540-8159.2012 .03335 . x$.

20. Voss A, Schulz S, Schroeder R, Baumert M, Caminal P. Methods derived from nonlinear dynamics for analysing heart rate variability. Phil Trans A Math Phys Eng Sci. 2009; 367(1887): 277-296. DOI: 10.1098/rsta.2008.0232.

21. Krstacic G, Krstacic A, Smalcelj A. The "Chaos Theory" and nonlinear dynamics in heart rate variability analysis: does it work in short-time series in patients with coronary heart disease? Ann Noninvasive Electrocardiol. 2007; 12(2):130-136. DOI: 10.1111/j.1542-474X.2007.00151.x.

22. Einstein A. Sobre la teoría de la Relatividad y otras aportaciones científicas. Madrid: Sarpe; 1983.

23. Rodríguez J, Prieto S, Flórez M, Alarcón C, López $\mathrm{R}$, Aguirre G, et al. Physical-mathematical diagnosis of cardiac dynamic on neonatal sepsis: predictions of clinical application. J Med Med Sci. 2014; 5(5): 102-108. DOI: http:/dx.doi.org/10.14303/ jmms.2014.070

24. Rodríguez J, Prieto S, Domínguez D, Correa C, Melo M, Pardo J, et al. Application of the chaotic power law to cardiac dynamics in patients with arrhythmias. Rev Fac Med. 2014; 62(4): 539546. DOI: http://dx.doi.org/10.15446/revfacmed. v62n4.43444.

25. Rodríguez J, Prieto S, Correa C, Soracipa Y, Aguirre G, Méndez L. Proportional entropy applied to the clinical diagnostic of cardiac dynamic: blind study with 600 holter. The 61 st Annual Conference of the 
Israel Heart Society in association with The Israel Society of Cardiothoracic Surgery. 2014.

26. Rodríguez J, Prieto S, Bernal P, Izasa D, Salazar G, Correa C, et al. Entropía proporcional aplicada a la evolución de la dinámica cardíaca. Predicciones de aplicación clínica. La emergencia de los enfoques de la complejidad en América Latina. Tomo I. Argentina: Comunidad Editora Latinoamericano; 2015: p.247-264.

27. Feynman R, Leighton R, Sands M. Leyes de Newton de la Dinámica. En R. Feynman, R. Leighton, M. Sands. Física. Wilmington: Addison-Wesley Iberoamericana, S. A.; 1987: p.9-1 - 9-14.

28. Feynman R, Leighton, R, Sands, M. Comportamiento cuántico. En R. Feynman, R. Leighton, M. Sands. Física. Wilmington: AddisonWesley Iberoamericana, S. A; 1987: p.37-1 - 37-16.

29. Rodríguez J, Bernal P, Álvarez L, Pabón S, Ibáñez $\mathrm{S}$, Chapuel N, et al. Predicción de unión de péptidos de MSP-1 y EBA-140 de plasmodium falciparum al HLA clase II Probabilidad, combinatoria y entropía aplicadas a secuencias peptídicas. Rev Inmunología. 2010; 29(3): 91-99.

30. Rodríguez J. Método para la predicción de la dinámica temporal de la malaria en los municipios de Colombia. Rev Panam Salud Pública. 2010; 27(3): 211-218.

31. Velásquez J, Prieto S, Correa $\mathrm{C}$, Dominguez D, Cardona DM, Melo M. Geometrical nuclear diagnosis and total paths of cervix cell evolution from normality to cancer. J Can Res Ther. 2015; 11(1): 98-104. DOI: 10.4103/0973-1482.148704.

32. Correa C, Rodríguez J, Prieto S, Álvarez L, Ospino B, Munévar A, et al. Geometric diagnosis of erythrocyte morphophysiology. J Med Med Sci. 2012; 3(11): 715-720.

33. Rodríguez J, Prieto S, Correa C, Bernal P, Puerta G, Vitery $\mathrm{S}$, et al. Theoretical generalization of normal and sick coronary arteries with fractal dimensions and the arterial intrinsic mathematical harmony. BMC Medical Physics. 2010; 10:1-6.

34. Rodríguez J, Prieto S, Correa C, Pérez C, Mora J, Bravo J, et al. Predictions of CD4 lymphocytes' count in HIV patients from complete blood count. BMC Med Phys. 2013; 13:3. DOI: 10.1186/17566649-13-3. 